Bull. Mater. Sci., Vol. 6, No. 6, December 1984, pp. 1087-1091. (C) Printed in India.

\title{
Surface modification of polyvinyl chloride towards blood compatibility
}

\author{
GEETHA KURIAN and CHANDRA P SHARMA \\ Department of Biomedical Engineering, Sree Chitra Tirunal Institute for Medical Sciences \\ and Technology, Trivandrum 695012 , India
}

MS received 29 January 1983; revised 6 September 1983

\begin{abstract}
The surface modification of medical grade polyvinylchloride (PVC) from various sources with the ionic bonding of polyelectrolyte is investigated by exposing the PVC sheets to $1 \%$ zephiran chloride for $10 \mathrm{~min}$ and then to $50 \mathrm{mg} \%$ solution of polyelectrolyte for $10 \mathrm{~min}$. Surface energy and platelet adhesion studies were carried out to demonstrate the suitability of our improved surface towards blood compatibility. Relative changes due to $\gamma$-irradiation are also discussed.
\end{abstract}

Keywords. Polyvinyl chloride; surface modification; blood compatibility

\section{Introduction}

Polyvinylchloride (PVC) with a wide choice of additives and ingradients such as plasticizers, fillers, stabilisers etc (Ownes and Read 1979) is being used for various biomedical applications. However the surface of the PVC may change depending upon the additives used. Since the prosthetic blood interaction is dependent on the nature of the surface, various surfaces may behave differently. Therefore, we have attempted in this paper to mention the modification of the medical grade PVC from different sources using polyelectrolyte towards improved blood compatibility.

Polyelectrolytes from synthetic poly (cis-1,4 isoprene) possess anticoagulant activity (van der Does et al 1979) and have been used for surface modification of polymers (Sederel et al 1983). Therefore we have chosen natural rubber, (Source Hevea Brasiliensis "Para Rubber") to obtain the polyelectrolyte for our studies. It seems the effect of surface modification with this method is independent of the pvc type and therefore appears quite promising for future investigations.

\section{Experimental work}

The PVC sheets of different sources are dipped in $0.1 \%$ soap solution. They are thoroughly cleaned with distilled water, finally rinsed with methanol and dried in vacuum oven. Polyelectrolyte (synthesised from natural rubber, van der Does et al 1979 ) is ionically bound on the various PVC sheets by first exposing to $1 \%$ Zephiran chloride for $10 \mathrm{~min}$ and then to polyelectrolyte $(50 \mathrm{mgm} \%$ in water) for $10 \mathrm{~min}$. The bare as well as the polyelectrolyte coated PVC sheets were irradiated at room temperature $\left(\mathrm{CO}^{60}\right.$ gamma source) with a constant dose rate of $0.25 \mathrm{M}$ rad (strength $843 / \operatorname{Rontgen} / \mathrm{hr} / \mathrm{m}$ ) for $4 \mathrm{hr} 30 \mathrm{~min}$. 
Inherent viscosity of the various bare PVC sheets was compared and the results are given in table 1. Variation of contact angle with time are also studied with a goniometer at room temperature using double distilled water as shown in figures 1-4.

\subsection{Blood coagulation studies}

Calf blood is used for evaluating the recalcification time under controlled $\mathrm{pH}$ and temperature with the PVC coated tubes. Glass tubes of $7.5 \mathrm{~cm}$ length and $1 \mathrm{~cm}$ diameter are coated with $10 \%$ solution of PvC in tetrahydrofuran. The tubes are dried at $60^{\circ} \mathrm{C}$ in a

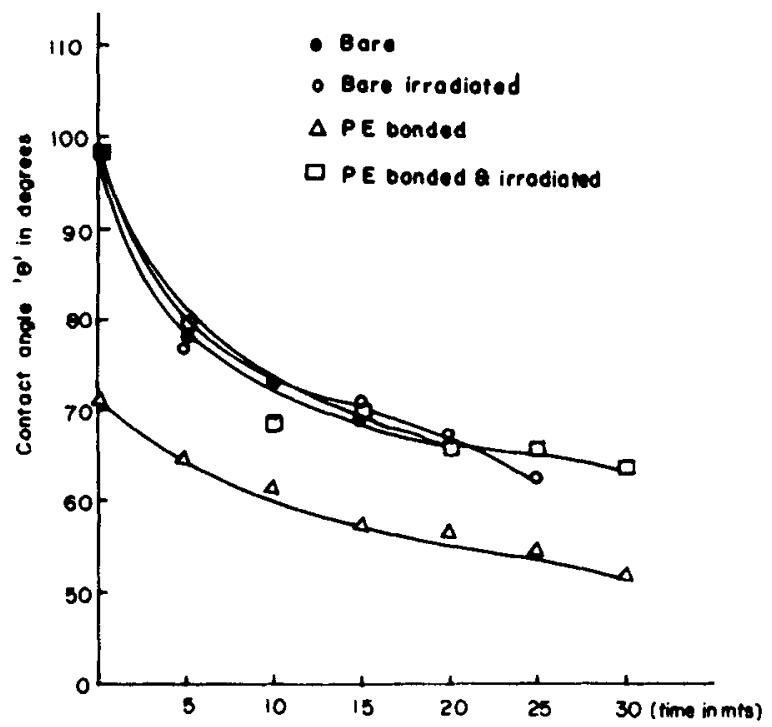

(1)

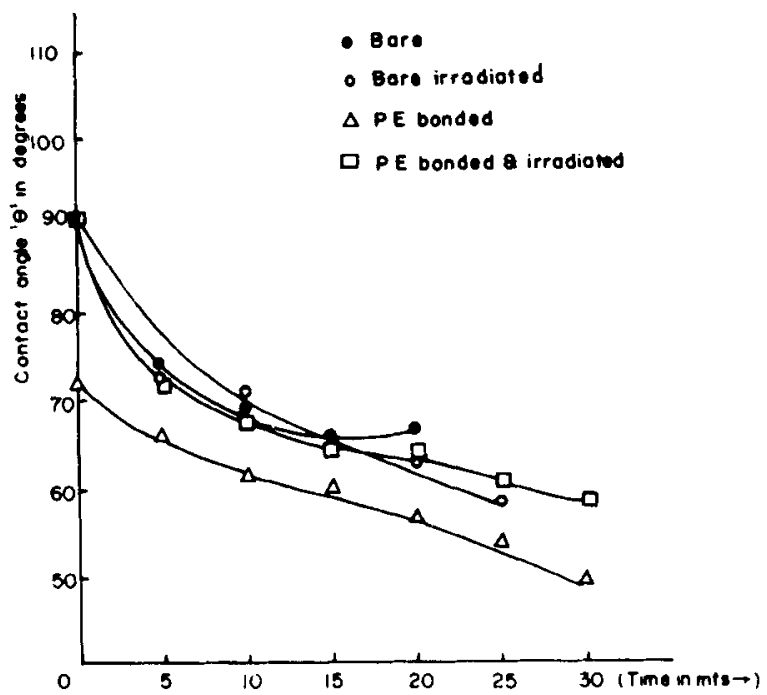

(2)

Figures 1-2. Change in contact angle with time on PVC surfaces 1. Bhor 2. SCTIMIST. 

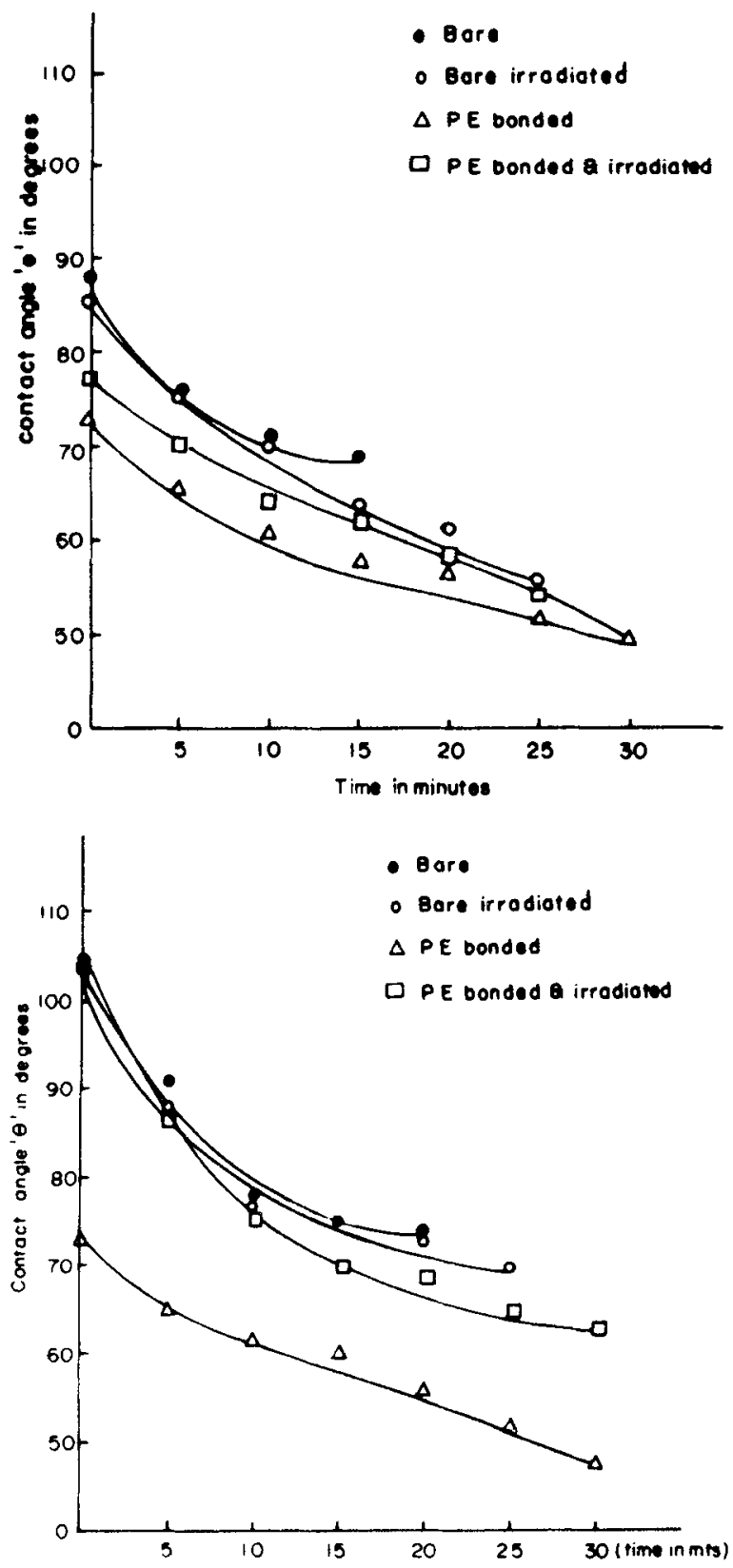

(4)

Figures 3-4. Change in contact angle with time on PVC surfaces 3. TUTA 4. Fenwal.

vacuum oven. The clotting time for the plasma is determined at $37^{\circ} \mathrm{C}$ (Austen and Rhymes 1975). The results are tabulated in table 2.

\subsection{Platelet adhesion on PVC surfaces: Preparation of platelet suspension}

Washed calf platelets are prepared and suspended in tyrode solution (Sharma and Chandy 1982) for adhesion studies. The washed PVC sheets are exposed to platelet 
Table 1. Inherent viscosity data of various PVC sheets.

\begin{tabular}{lc}
\hline PVC sheets & Viscosity (dl/g) \\
\hline I & 1.27 \\
II & 1.03 \\
III & 1.01 \\
IV & 1.08 \\
\hline
\end{tabular}

I-Bhor Industry India Product for sctimst. 1I-Polymer Technology Division Product of our Institute for blood bag applications, submitted by S N Pal, sctimST. III-Tuta Industry Australia, blood bag. IV-Fenwal, Travenol Inc. (USA), blood bag.

Table 2. Plasma recalcification time

\begin{tabular}{lc}
\hline Surfaces & $\begin{array}{c}\text { Plasma recalcification } \\
\text { time (sec) SD } \\
(n=5)\end{array}$ \\
\hline Plain glass & $140 \pm 2$ \\
I & $187 \pm 5$ \\
II & $166 \pm 11$ \\
III & $340 \pm 3$ \\
IV & $198 \pm 20$ \\
\hline
\end{tabular}

I, II, III and IV are as noted in table 1.

Table 3. Platelet adhesion on various PVC sheets.

\begin{tabular}{lccccc}
\hline & \multicolumn{2}{c}{ Bare PvC sheets } & & \multicolumn{2}{c}{ Polyelectrolyte coated Pvc sheets } \\
& $\begin{array}{c}\text { Before } \\
\gamma \text {-irradiation } \\
\text { PVC sheets }\end{array}$ & $\begin{array}{c}\text { After } \\
(n=10)\end{array}$ & $\begin{array}{c}\gamma \text {-irradiation } \\
(n=10)\end{array}$ & $\begin{array}{c}\gamma \text {-irradiation } \\
(n=10)\end{array}$ & $\begin{array}{c}\text { Before } \\
\gamma \text {-irradiation } \\
(n=10)\end{array}$ \\
\hline I & $5 \cdot 8 \pm 0.8$ & $4.9 \pm 0.7$ & $3.6 \pm 0.5$ & $4.2 \pm 0.4$ \\
II & $5 \pm 0.8$ & $4.9 \pm 0.7$ & $3.6 \pm 1.2$ & $4.2 \pm 0.5$ \\
III & $5 \pm 1.0$ & $4.9 \pm 0.7$ & $3.6 \pm 0.53$ & $3.8 \pm 1.0$ \\
IV & $5.8 \pm 0.8$ & $5 \pm 0.7$ & $3.6 \pm 1.2$ & $4.4 \pm 0.6$ \\
\hline
\end{tabular}

I, II, III and IV are as noted in table 1.

suspension for $1 \mathrm{hr}$, rinsed with phosphate buffer of $\mathrm{pH} 7.4$ under uniform flow for about $3 \mathrm{~min}$ and the platelets are fixed with Coomassie Blue $\mathrm{G}$. The number of platelets adhered to the PVC sheets are accounted using an optical microscope. Different fields are read randomly and averaged in a similar fashion for all samples. The results are given in table 3.

\section{Results and discussion}

Platelet adhesion and contact angle studies were carried out on bare as well as on polyelectrolyte coated PVC surfaces to investigate the effect of polyelectrolyte coating on PvC towards blood compatibility. 
It seems when polyelectrolytes are on the surface the material is improved towards blood compatibility. Platelet adhesion is relatively less as indicated in table 3 . Although they are different before modification as indicated by our plasma recalcification time (table 2) and the change in contact angle with time (figures 1-4), the contact angle becomes relatively more stable and the behaviour of the various PVC sheets becomes alike (figures 1-4). After irradiation the effect of polyelectrolyte is no longer obvious as shown in figures 1-4. It becomes a part of the PVC structure such that leaching components do not have any additional resistance as it was before irradiation. Therefore we suggest that PVC surfaces can be improved towards blood compatibility like polyelectrolyte as described above, provided the material is already sterilised by $\gamma$ irradiation, since for long term applications ionic bonding of polyelectrolytes with PVC surface may not be stable enough. This modification is suggested for all short term medical applications of PVC coming in contact with blood such as oxygenators and blood bags etc. No adverse change in platelet adherance is observed on modified surfaces if autoclaving sterilization (Sharma and Nirmala 1983) is used instead of $\mathrm{CO}^{60}, \gamma$-irradiation.

\section{Acknowledgement}

The authors appreciate the help received from Dr V N Krishnamurthy of Vikram Sarabhai Space Centre, Trivandrum for ${ }^{60} \mathrm{Co} \gamma$-irradiation of our samples, Dr A V Lal for providing calf blood, Mr S N Pal for the PvC samples and Mr Thomas Chandy and Mrs M K Sheela for help.

\section{References}

Austen D E G and Rhymes I L 1975 A laboratory manual of blood coagulation (Oxford: Blackwell Scientific Publications) p. 35 .

Owen B D and Read R L 1979 J. Polym. Sci., Polym. Chem. Ed. 172719

Sederel L C, van der Does E, Evverman B J, Bantjes A, Kluft C and Kempen H J M 1983 Biomaterials 43 Sharma C P and Chandy T 1982 J. Colloid Interface Sci. 89479

Sharma C P and Nirmala N V 1983 Polyvinyl chloride compatibility at National Seminar on Recent developments in Polymer Science, AC College Campus, Madras, December 22 and 23rd, Proceedings to follow in honour of Prof. M Santappa

van der Does L, Bengeling T, Frochling P E and Bantjes A J 1979 Polym. Sym. 66337 